Document downloaded from:

http://hdl.handle.net/10251/56289

This paper must be cited as:

López Sánchez, J.; Zöllner, J.; Atungsiri, S.; Stare, E.; David Gomez-Barquero (2014). Technical Solutions for Local Service Insertion in DVB-NGH Single Frequency Networks. IEEE Transactions on Broadcasting. 60(2):293-301. doi:10.1109/TBC.2014.2322502.

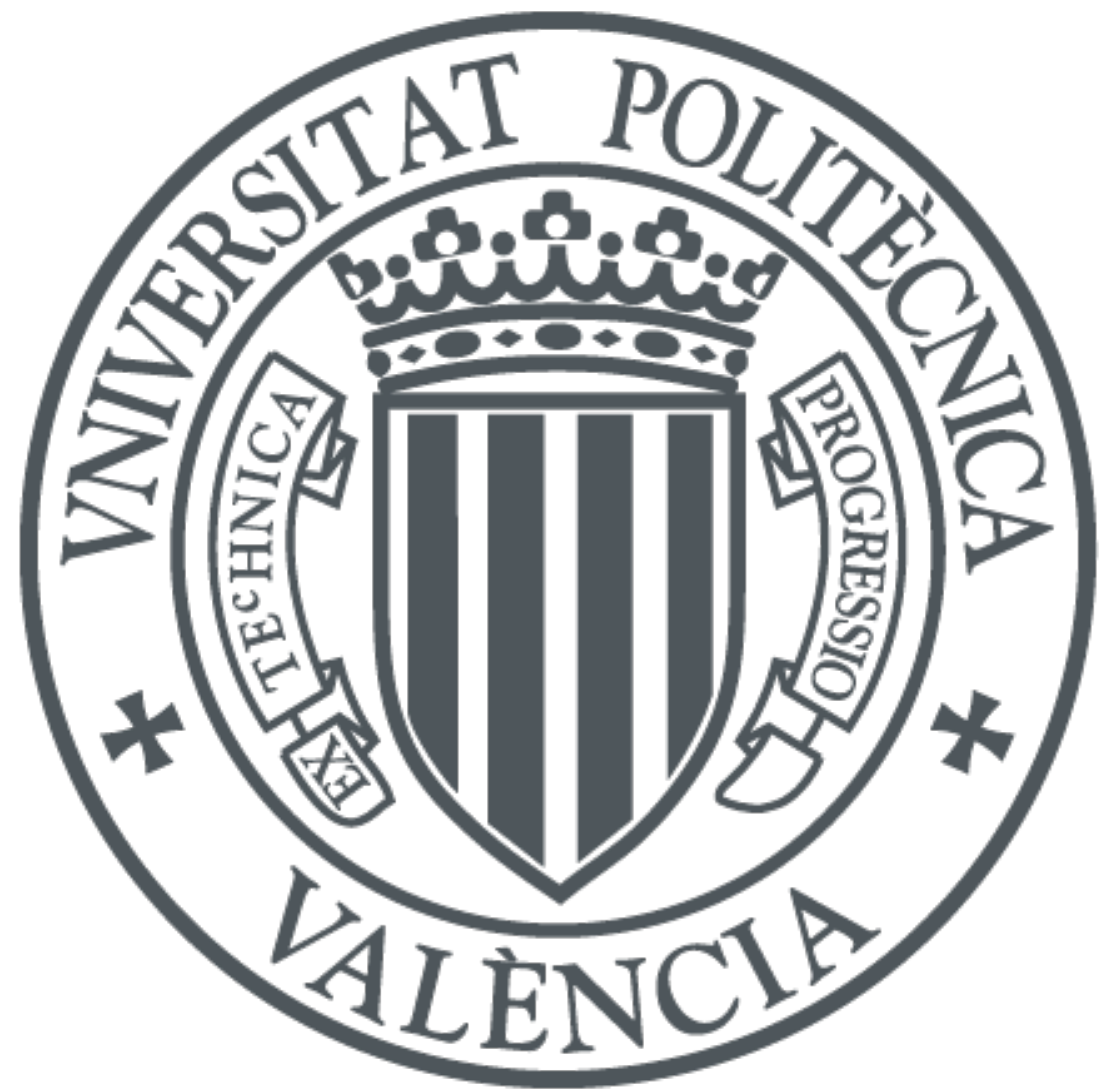

The final publication is available at

http://dx.doi.org/10.1109/TBC.2014.2322502

Copyright Institute of Electrical and Electronics Engineers (IEEE)

Additional Information 


\title{
Technical Solutions for Local Service Insertion in DVB-NGH Single Frequency Networks
}

\author{
Jaime López-Sánchez, Jan Zöllner, Samuel Atungsiri, Erik Stare and David Gómez-Barquero
}

\begin{abstract}
Current Digital Terrestrial Television (DTT) networks make use of either Single Frequency Networks (SFN) or Multi Frequency Networks (MFN). These network topologies are not ideally suited for delivery of both global and local services in an efficient way. MFNs enable the efficient transmission of local services but need a significant amount of frequency spectrum. The basic principle of SFNs is that all transmitters radiate the same signal synchronized in time and frequency, therefore are especially suitable for global services due to mutual support of the signal by multiple transmitters. Without violating the SFN principle, local services meant to address sub-regions of an SFN must therefore be transmitted throughout the whole network, causing inefficient distribution of local services. This paper describes the complementary techniques adopted by the next generation mobile broadcasting standard DVB-NGH (Digital Video Broadcasting - Next Generation Handheld) for providing global and local contents in SFN topologies: Hierarchical Modulation (H-LSI) and Orthogonal Local Services Insertion (O-LSI) techniques. H-LSI uses hierarchical modulation to transmit local services on top of the global services in areas close to the transmitters, by transmitting the local services in the Low Priority (LP) stream and the global services in the High Priority (HP) stream. The O-LSI scheme specifies groups of OFDM subcarriers in specific OFDM symbols for the exclusive use of particular transmitters to transmit local services. For both techniques, the transmission of local content through the whole SFN network can be scheduled in a way that different local areas do not interfere with each other. In addition to the description of both H-LSI and O-LSI schemes, the applicability of these approaches in terms of network topologies, implementation issues and performance evaluation are analyzed.
\end{abstract}

Index Terms - DVB-NGH, Hierarchical modulation, Local services, Orthogonal local services insertion, Single Frequency Networks (SFN).

\section{INTRODUCTION}

$\mathrm{T}$ elevision (TV) services transmitted in a Digital Terrestrial Television (DTT) network can be classified depending on their target area. Most of the services target all the users throughout the whole network, and are usually called global

Manuscript received July 09 of 2013; revised March 24 of 2014 and accepted XX of 2014. Jaime López-Sánchez and David Gómez-Barquero are with the iTEAM Research Institute, Universitat Politècnica de València, Valencia, Spain (\{jailosan, dagobar\}@iteam.upv.es). Jan Zöllner is with the Institute for Communications Technology (IfN) of Technische Universität Braunschweig, Braunschweig, Germany (zoellner@ifn.ing.tu-bs.de). Samuel Atungsiri is with Sony Semiconductor Design Centre, Basingstoke, United Kingdom, (samuel.atungsiri@eu.sony.com). Erik Stare is with Teracom AB, Stockholm, Sweden (erik.stare@teracom.se). services. In contrast, some services are only relevant for viewers in a certain sub-region of the network and they are referred to as local services.

One of the main advantages of DTT networks is the possibility of deploying SFNs by the use of Orthogonal Frequency Division Multiplexing (OFDM) with a sufficiently long Cyclic Prefix (CP). The receiver usually receives multiple signals coming from different SFN transmitters with different channel attenuations and time delays, which exhibits a severe artificial multipath effect. All the signals from the different transmitters should arrived inside the CP interval in order to be considered constructive to the wanted signal.

SFNs are ideally suited for global services because of the need of a single frequency channel and due to the mutual support of the signals from the different transmitters, the socalled SFN gain [1]. However, if local services are transmitted, they have to be transmitted across the whole network, including regions where they are not required. This leads to a significant waste of capacity if the proportion of local content is large. On the other hand, using a MFN approach, the full channel capacity is available for the content transmitted within each cell. The main drawback is that more spectrum is required compared to the SFN approach.

An ideal solution to transmit global and local content in SFN networks should retain all SFN advantages for global services. The transmission of local services should be spectrally efficient and using any subset of sites of the network, while their coverage area (Local Service Area, LSA) is restricted to the specific areas where local content is to be consumed. In order to achieve this, the SFN principle has to be violated partially, e.g. for a short period of time or a limited frequency range. The main problem is that different local services transmitted within a single frequency cause interference. Thus, in areas where the signals of two or more sites transmitting different local services are strong, successful reception of local services may not be possible. However, for local services a reduced coverage area compared to global services may be acceptable for some use cases (e.g. urban areas), although for some uses cases the required coverage can be the same as for global services.

The current state-of-the art DTT system, DVB-NGH (Digital Video Broadcasting - Next Generation Handheld) [2], will allow exploring the viability of inserting local services in SFNs in a way that has not been possible before. DVB-NGH is the handheld evolution of the second generation digital terrestrial TV standard DVB-T2 (Terrestrial $2^{\text {nd }}$ Generation) [3], and one of the main technical innovations introduced with respect to DVB-T2 is the efficient 

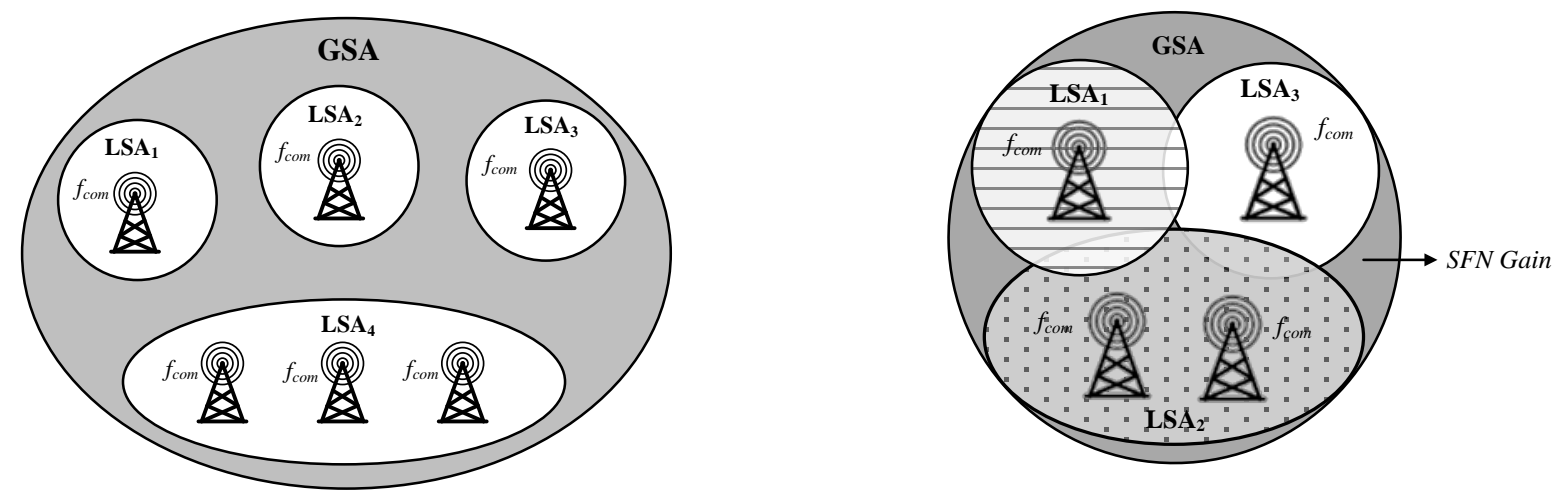

Fig. 1. Coverage areas for global and local services in a SFN using H-LSI (left) and O-LSI (right). All transmitters employ the same frequency.

provisioning of local content in SFNs. DVB-NGH has adopted two complementary techniques with small network overhead to transmit local content in SFNs, known as Hierarchical and Orthogonal Local Service Insertion (H-LSI and O-LSI, respectively) [4]. The first technique uses Hierarchical Modulation (HM), which generates each QAM symbol from two bit streams with different robustness levels (global content is transmitted within the so-called High Priority (HP) bit stream, whereas the local content is inserted into the Low Priority (LP) stream) [5] [6]. HM was adopted for the first time for DVB-T (Terrestrial) [7], and it was also adopted for the mobile broadcasting system Media FLO [8] and DVB-SH (Satellite to Handhelds) [9], although it has never been commercially deployed yet. With O-LSI technique, a set of OFDM (Orthogonal Frequency Division Multiplexing) subcarriers within the NGH frame structure are allocated to transmit local services. The transmitters of each LSA transmit local content using a subset of these sub-carriers. This concept is similar to the auxiliary stream insertion specified in the DVB-T2 transmitter signature standard [10]. O-LSI is a novel technique for which no previous studies or performance results are available in the literature.

The main contribution of this paper is the description of the concept and implementation aspects of both H-LSI and O-LSI technical solutions adopted for DVB-NGH to insert local content in SFNs and the evaluation of their coverage performance and their transmission capacity gain.

The rest of this paper is structured as follows. Section II describes the network topologies for H-LSI and O-LSI. Section III and Section IV describe the main characteristics use of the H-LSI and O-LSI techniques. Section V presents coverage performance evaluation results obtained by means of physical layer simulations. Section VI discusses the transmission capacity gain achieved with each technique. Finally, the paper is concluded in Section VII.

\section{NETWORK TOPOLOGIES FOR LSI IN DVB-NGH}

The principle of SFNs with global and local services for $\mathrm{H}$ LSI and O-LSI is shown in the Fig. 1. All transmitters employ a common frequency, $f_{\text {com, }}$, to transmit both global and local services. For global services, a coverage gain within the Global Services Area (GSA) is achieved due to a statistical gain by exploiting the signal diversity and a power gain by the combination of the received signal strengths (SFN gain). Local services are only provided in the three depicted LSAs.

For H-LSI, the coverage of the local services is limited to the areas surrounding the transmitters. This may be acceptable for some use cases (e.g. when the transmitter is located within a city). In this case, signals from different LSAs act as interference if the insertion took place at identical time instances. A solution to avoid interferences between LSAs is to time share the hierarchical transmission mode, e.g. on a frame-by-frame basis. Time sharing slots can be reused between transmitters that are sufficiently far apart. Time sharing leads to a reduced capacity available for local content that can be inserted at each LSA.

The main advantage of O-LSI is that it is possible to provide local services across the whole network with basically the same coverage as the global services, not necessarily only in the vicinity of the transmitters as the first approach of $\mathrm{H}$ LSI. Hence, this technique is suitable, for example, for local news or advertising as temporal window in a global service. The right side of Fig. 1 shows the coverage level for global and local services in an SFN with O-LSI. In the overlapping zones between adjacent LSAs, global services experience an SFN gain whereas local services do not, but the receivers can decode more than one local service.

\section{Hierarchical MOdUlation FOR LOCAL SERVICES INSERTION IN DVB-NGH SFNS}

\section{A. Concept}

DVB-NGH supports hierarchical 16QAM and 64QAM modulation for the insertion of local services, where the global services employ a QPSK or 16QAM modulation, and the transmitters inserting local services add an additional QPSK constellation on top of the global QAM constellation, containing the local service. For the global service, the hierarchically modulated QAM symbols "look" like noise, requiring an increase in CNR (Carrier-to-Noise Ratio). This effect diminishes with distance from the local service inserting transmitter as shown in Fig. 2. Since the local service is mapped to the low priority bits of the constellation, the effective CNR of the local service is smaller compared to the global service [6]. The required CNR for successful reception of the local stream can however be adapted by choosing a smaller code rate of the forward error correction (FEC) code for the local service compared to the global service. 

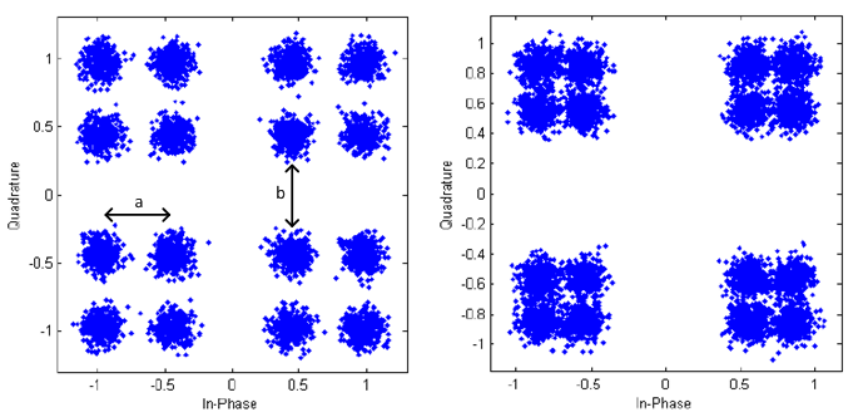

Fig. 2. Received signal constellation in a network comprising two transmitters, one transmitting the global service only using QPSK, the other one transmitting both global and local stream using hierarchical 16-QAM; left: receiver is close to the local transmitter, right: receiver is distant from the local transmitter and near to the non-hierarchical transmitter.

The HM causes inter-layer interference, since the LP stream acts as noise to the HP stream (and vice versa), causing a coverage reduction of both the local and global services. The robustness of both LP and HP streams can be adjusted by means of the HM parameter $(\alpha)$ that describes the ratio of the smallest distance between the constellation points carrying different HP bits $(b)$ to the distance between the LP bits $(a)$, as shown in Fig. 2 [2]. In DVB-NGH, the allowed values for $\alpha$ are 1, 2 and 4 for 16QAM, and 1 and 3 for 64QAM. For high values of $\alpha$, the degradation of the HP stream is practically negligible, but the robustness of the LP stream is significantly reduced. The selection of $\alpha$ is therefore a trade-off between the coverage reduction of the global services and the coverage achieved for the local services.

A second penalty for global services decoding arises from the violation of the SFN principle due to the symbols emitted from the all transmitters are not the same. Since the transmitters with global and local services transmit different complex values in all OFDM carriers that carry hierarchically modulated local services, compared with the transmitters with global services only. This causes imperfect equalization in the receiver and causes a performance penalty for the global and local services that depends on the channel characteristics at the receiver. The penalty can be reduced with an iterative equalization and decoding scheme, called Iterative Sliced Decoding (ISD) [11].

\section{B. Implementation Aspects}

DVB-NGH re-uses the PLP (Physical Layer Pipe) feature of DVB-T2. A PLP is a logical channel carrying one or multiple services. Each PLP may use a different modulation scheme and code rate within the same transmission channel to meet different reception conditions (e.g. portable indoor or roof-top

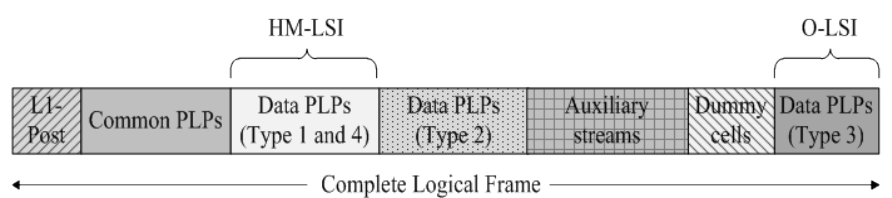

Fig. 3. Structure of the DVB-NGH LF with the different types of PLPs.

reception). In DVB-T2, each transmission frame comprises two types of PLPs, known as Type 1 and Type 2 [3]. PLPs of Type 1 are transmitted in a single burst (slice) within each frame, whereas PLPs of Type 2 are transmitted in multiple subslices within each frame. In DVB-NGH, two new types of PLPs are defined for local service insertion in SFNs, known as Type 3 and Type 4, which are used for O-LSI and H-LSI, respectively [2]. Fig. 3 shows the NGH logical frame (LF) structure showing the different types of PLPs. H-LSI PLPs are transmitted on top of data PLPs of Type 1, being mapped after the common PLPs. The O-LSI PLPs are transmitted at the end of the frame, after data PLPs of Type 2. Auxiliary streams or padding sub-carriers may exist in-between Type 2 and Type 3 PLPs.

With H-LSI the local and global service bits are processed separately in two stages, both containing the typical blocks of the DVB-NGH signal generation as shown in Fig 4. However, the processing path of the local PLP comprises a special burst builder, which groups the coded local service bits of an integer number of FEC frames and inserts a 64 bit synchronization header at the beginning, building a local service frame. The synchronization header carries the signaling information for local PLP decoding. The value of the hierarchical parameter $\alpha$ and the ID of the global PLP carrying the local PLP are signaled in the physical layer (L1) signaling, since this information is required to extract the local stream.

Before the hierarchical QAM mapping takes place, local pilots are inserted that are required for channel estimation and equalization in the receiver [11]. Local pilots are hierarchically modulated on top of the global QAM symbols with the same spacing as the global PLPs. However, the local pilots are shifted by one carrier compared to the global pilots to avoid distortion of the global pilots. Afterwards, both streams are jointly mapped by the QAM mapper to the most significant bits MSBs and least significant bits LSBs of the hierarchical QAM constellation. If constellation rotation is used for the global service PLP, the same rotation angle is applied to the hierarchically modulated QAM symbols.

For successful decoding of the local stream, the global

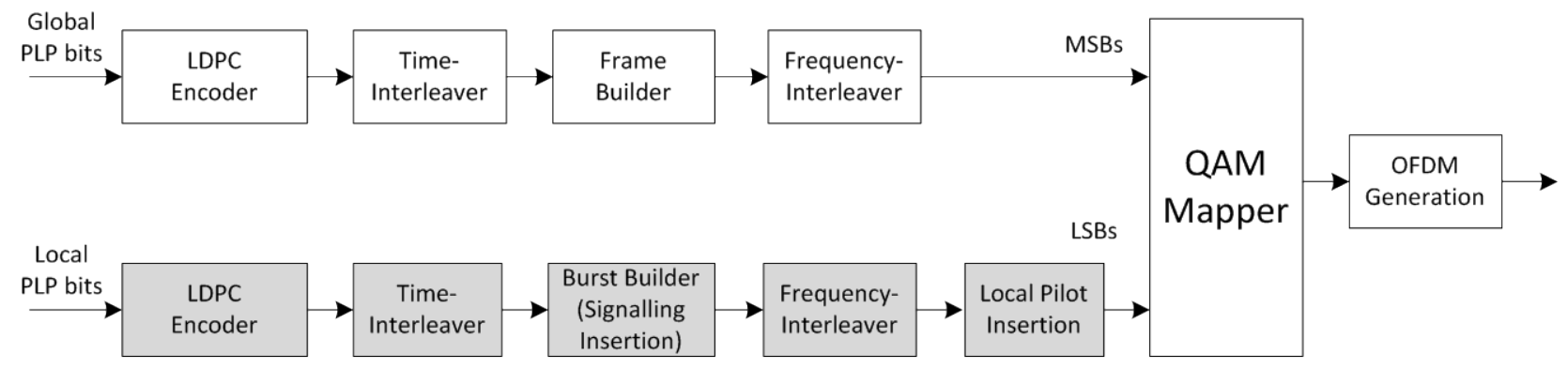

Fig. 4. H-LSI transmitter block diagram 


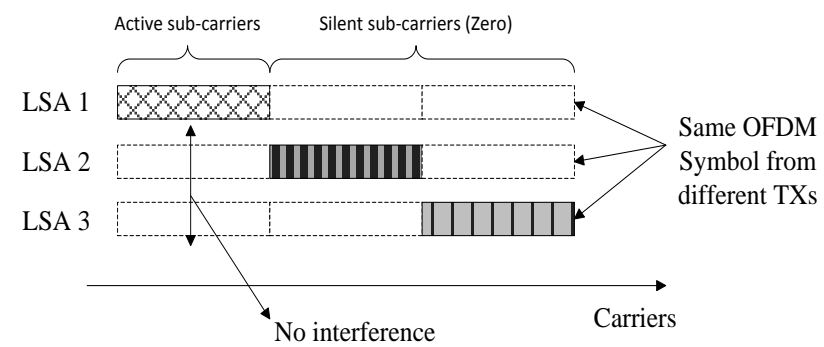

Fig. 5. General concept of the O-LSI technique. Each local transmitter allocates a specific set of OFDM sub-carriers to its local services.

service needs to be subtracted from the received signal. This operation requires knowledge of the Channel Transfer Function (CTF) between the local transmitter and receiver, due to the violation of the SFN principle. As the initial decoding of both global and local services is distorted since neither the local nor the global streams for subtraction from the received signal are known, the decoding is carried out in an iterative manner by means of an ISD process [11]. In the first iteration, imperfect reception of the global service is performed, and the local service is decoded by subtracting the global service from the received signal. If decoding is not fully successful, a second iteration can be carried out, and so on, improving the both global and local performance in each iteration.

The H-LSI technique is neither compatible with nonuniform QAM constellations nor with the Peak-to-Average Power Ratio (PAPR) reduction scheme Active Constellation Extension (ACE).

\section{ORTHOGONAL LOCAL SERVICES INSERTION IN DVB-NGH SFNS}

\section{A. Concept}

With O-LSI a set of dedicated OFDM sub-carriers on dedicated OFDM symbols are reserved for the transmission of local services. Within the same OFDM symbol, the transmitters of different LSAs employ a different subset of sub-carriers to broadcast local services, whereas the same OFDM sub-carriers used by the other transmitters are unused. The orthogonality obtained by using dedicated carriers for each local service ensures that no interference between adjacent transmitters occurs (Assuming correct frequency synchronization between SFN transmitters). Fig. 5 shows the general concept of the O-LSI technique for the insertion of local services in an SFN with three LSAs. For the sake of clarity, the picture shows the allocated data subcarriers in one OFDM symbol before frequency interleaving. After frequency interleaving, each set is spread across the complete bandwidth to achieve high frequency diversity, still avoiding interference between transmitters of different LSAs. However, similar to other OFDM systems, the frequency offsets such as Doppler Effect, can affect this orthogonality, resulting in inter-carrier interference (ICI) due to power leakage among subcarriers. In this case, the performance of global and local services in mobile reception is similar to the performance DVB-NGH in conventional SFN topology [12] and depends on the velocity of the receiver and the robustness of the transmission mode.

The main advantage of the O-LSI technique is that no interference between transmitters from different LSAs occurs, because the inserted local content is orthogonal to each other. Furthermore, LSAs separated by a long distance can reuse the same sub-carriers following a spatial reuse pattern. This way, local services can potentially have the same coverage area as global services, allowing receiving at least one local service at any point in the network. Furthermore, the insertion of local services does not affect the coverage area of the global services. The drawback is the reduction of the capacity available for global services, since the local services are not transmitted on top of the global services. With O-LSI, global and local services share all available OFDM data sub-carriers. O-LSI allows for a power boosting of the OFDM sub-carriers devoted to local services in order to compensate the unmodulated carriers of others LSAs keeping a constant OFDM symbol power over time. This power boosting can either be translated into a capacity increase using a transmission mode with higher spectral efficiency (higher code rate and/or higher modulation order) or can alternatively be used to improve the coverage area of the local services.

\section{B. Implementation Aspects}

The payload data using O-LSI is transmitted as Type 3 PLP after any preceding Type 1 and Type 2 PLPs in specific OFDM symbols, as shown in Fig. 5. All Type 3 PLP data in a transmission frame is transmitted by a number of consecutive OFDM symbols with the first and the last O-LSI having a denser pilot pattern.

The orthogonality among different local content is obtained by dividing the available number of data sub-carriers in each O-LSI symbol into the number of local services areas parts, $n_{L S A}$. Only one part is then transmitted from a particular transmitter. When all O-LSI data cells have been introduced, frequency interleaving is performed symbol by symbol.

In a similar way to H-LSI, O-LSI PLPs require dedicated
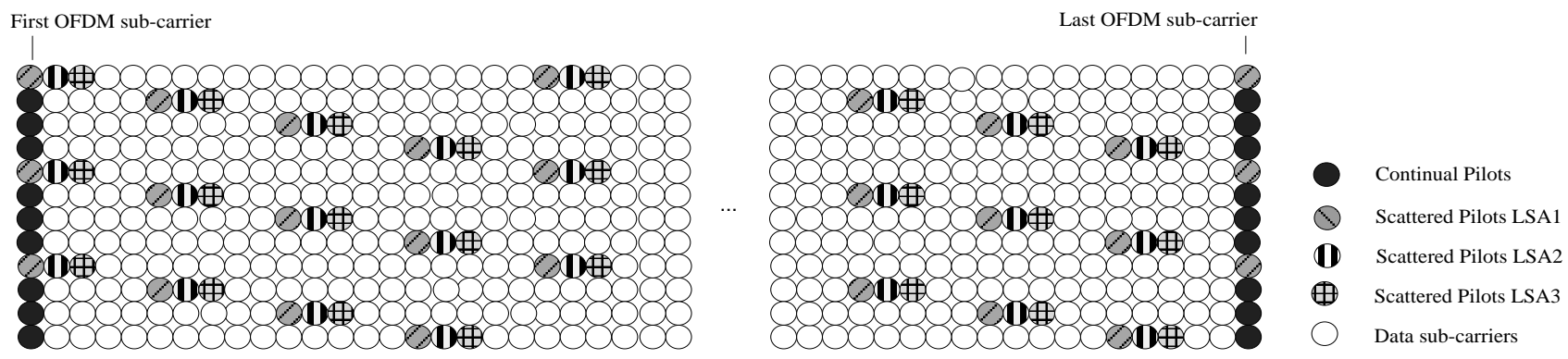

Fig. 6. Pilot pattern with three LSAs based on PP7 
TABLE I.

MINIMUM CNR (dB) FOR SUCCESSFUL DECODING OF HP STREAM. LDPC 16200, PP4.

\begin{tabular}{ccccc}
\hline \hline $\begin{array}{c}\text { Modulation and Code } \\
\text { rate (MODCOD) of } \\
\text { the HP stream }\end{array}$ & Channel Model & $\begin{array}{c}\text { Non-HM } \\
(\alpha=\text { inf })\end{array}$ & $\begin{array}{c}\text { HM } \\
\alpha=1\end{array}$ & $\begin{array}{c}\text { HM } \\
\alpha=3\end{array}$ \\
\hline QPSK CR 7/15 & Rayleigh P1 & 2 & 4.2 & - \\
& TU6@27Hz & 4.2 & 10 & - \\
QPSK CR 2/3 & Rayleigh P1 & 3.8 & 8 & - \\
& TU6@27Hz & 5.3 & 16 & - \\
16-QAM CR 7/15 & Rayleigh P1 & 7.8 & 9.8 & 8.3 \\
& TU6@27Hz & 11.2 & 13.2 & 11.8 \\
\multirow{2}{*}{ 16-QAM CR 2/3 } & Rayleigh P1 & 12.3 & 14.8 & 12.9 \\
& TU6@27Hz & 16 & 19 & 16.8 \\
\hline \hline
\end{tabular}

pilots for channel estimation. These additional pilots reduce the useful data capacity depending on the number of LSAs in the SFN. The continual pilots, e.g. used for frequency synchronization, are the same for all transmitters in the network. However, as shown in Fig. 6, scattered pilots must be inserted for each LSA each being frequency shifted by one OFDM sub-carrier, such that the different PPs are orthogonal. Since the scattered PP is repeated for each LSA, the densest patterns PP1 and PP2 are not available for O-LSI to avoid extensive pilot overhead.

The reserved O-LSI data and pilot sub-carriers in each LSA are transmitted with an amplitude boosting factor equal to $\sqrt{ } n_{\text {LSA }}$, followed by a normalization factor $K$. The value of $K$ is chosen such that the O-LSI starting and closing symbols have the same expected average power as the preamble P2 symbol, which carriers the L1 signaling. The power level of any intermediate symbols is slightly lower due to a decreased pilot density. The O-LSI specific signaling is transmitted as part of L1 signaling [2].

\section{Coverage Performance Results}

The simulation results presented in this section have been obtained using an end-to-end DVB-NGH physical layer simulation platform. This platform was validated in the DVBNGH standardization process and is constantly evolving and adapting to the techniques developed in the framework of the DVB terrestrial standards. For global services, all signals arrived within the $\mathrm{CP}$ are considered as constructive contribution to the wanted signal, creating an SFN gain, while the signals exceeding the $\mathrm{CP}$ are considered destructive interference. However, for some cases, the increase of the CNR due to the SFN gain does not guarantee an improvement in the reception quality [13][14]. This power gain is beneficial for receivers operating under diffuse multipath or low CNR conditions (e.g. Rayleigh P1 and TU6 channel models), while in those scenarios with high CNR and strong Line of Sight (LOS) the presence of SFN echoes degrades the reception quality (e.g. AWGN and Rice F1 channel models) [15].

\section{A. H-LSI Coverage Performance}

The coverage performance of H-LSI has been obtained considering two neighboring SFN transmitters: one delivering HM (global + local stream) signal and the other delivering the

global stream only. The Power Imbalance (PI) between transmitters describes the power difference in $\mathrm{dB}$ between the signal received from the non-HM transmitter and the signal received from the HM transmitter. These results can be extended to more than two neighboring LSAs considering the signals from other LSAs as interference for the selected LP stream. For the simulations, two uncorrelated static Rayleigh P1/P2, or two uncorrelated TU6 fading channel models with an additional delay between the channel impulse responses have been introduced, in order to emulate different propagation channel characteristics from the two transmitters. The selected Doppler frequency in TU6 channel is $27 \mathrm{~Hz}$, which corresponds to $60 \mathrm{~km} / \mathrm{h}$ for a RF carrier of $500 \mathrm{MHz}$. A time interleaving duration of $58 \mathrm{~ms}$ has been selected, which is enough to overcome the mobile reception impairments in 8 $\mathrm{MHz}$ bandwidth channel.

\section{1) Coverage degradation of global services}

With H-LSI, the available bitrate for global services can be the same that the classic SFN approach without local services insertion. However, the hierarchically modulated local service adds a coverage penalty to the global services due to the interlayer interference. In order to keep the same coverage level of global services offer by the classic SFN without LSI, a higher MODCOD configuration can be used, at expensed of reduce the available bitrate.

The coverage penalty can be expressed by means of a reduced effective CNR for the global services depending on the value of $\alpha$ [5]. Table I shows the minimum CNR required for successful decoding ${ }^{1}$ of the global stream for different values of $\alpha$ when a signal from the HM transmitter is received only. It shows the worst case values, since the interlayer interference decreases with greater distances from the local services inserting transmitter. In this case, the penalty for the global stream corresponds to the difference between the minimum CNR for non-HM $(\alpha=\infty)$ and the CNR required for the HP stream of the HM transmission mode. This penalty is basically generated by the displacement of the constellation point introduced by the over modulation of the LP stream and depends on the modulation scheme, the code rate and the value of $\alpha$ selected. Naturally, without LSI, (i.e. $\alpha=\infty$ ), no penalty occurs.

The second penalty due to the breakup of the SFN principle cannot be calculated in such general way since it depends on the location of the receiver within the SFN area and its channel characteristics. Fig. 7 depicts the overall penalty on the global stream decoding, taking the interlayer interference of $\mathrm{HM}$ and the power imbalance between the received signals in account. As reference, the dashed lines show the performance of non-HM 16QAM CR 7/15 emitted from both SFN transmitters in Rayleigh P1 and TU6 channels. Compared with these references, the penalty is manly generated by interlayer interference (around $2 \mathrm{~dB}$ for $\alpha=1$, corresponding with the results shown in Table I) in the vicinity of the HM transmitter, but is getting larger when the receiver moves toward the middle of the SFN area. The overall penalty reaches the highest value just in-between both transmitters, which can be up to $3 \mathrm{~dB}$ for portable and mobile reception with $\alpha=1$. The penalty decreases in areas where the received

\footnotetext{
${ }^{1}$ The quality criterion used in this paper is a BER of $10^{-7}$ after $\mathrm{BCH}$, which is comparable to a FER of $10^{-5}$ after BCH.
} 


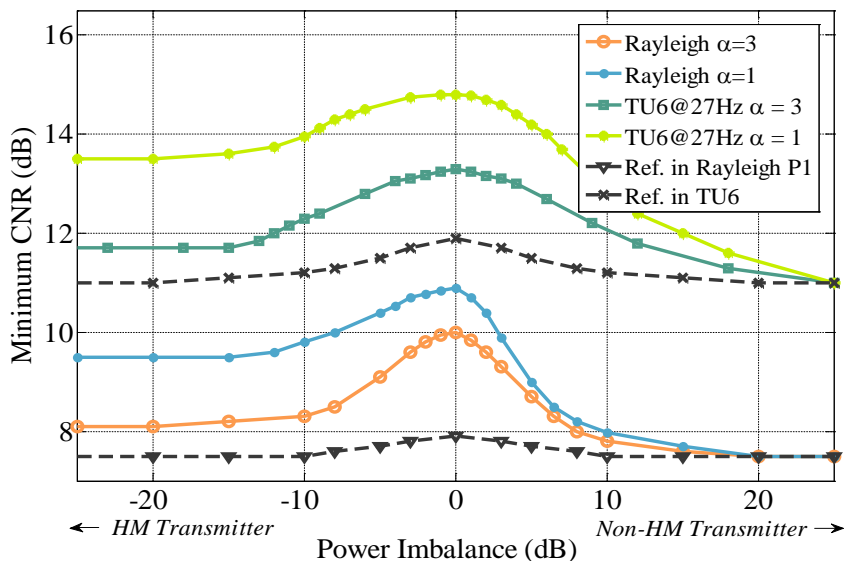

Fig. 7. Performance of global services using H-LSI. Hierarchical 64QAM CR 7/15 in Rayleigh and TU6 channels. The dashed lines are the performance of classic 16QAM 7/15.

power from the non HM transmitter is dominant, becoming negligible in the vicinity of this.

\section{2) Performance of local services}

The performance of local stream decoding with one ISD iteration in the Rayleigh P1 and TU6 channel is shown in Fig. 8 for different locations of the receiver between the HM and non-HM transmitters. Successful decoding of the local services with ISD is possible even in areas with higher signal power level from the non HM transmitter compared to the HM transmitter (i.e. PI >0) if the CNR is very high. As the distance from the HM transmitter increases, the required CNR for successful decoding also increases. Taking as reference an increase of maximum $3 \mathrm{~dB}$ in the minimum CNR required at the vicinity of the HM transmitter, we can observe that the maximum power imbalance permitted for successful decoding of local services in the Rayleigh P1 channel is $3 \mathrm{~dB}$ and $0 \mathrm{~dB}$ for H-LSI with $\alpha=1$ and $\alpha=3$, respectively. Lower values of power imbalance are allowed for correct decoding of local services in TU6 channel.

Compared with the performance at the vicinity of HM transmitter in the Rayleigh P1 channel of 64QAM CR 7/15, which is the overall modulation scheme used for local services, a penalty of $2 \mathrm{~dB}$ and $7 \mathrm{~dB}$ in the minimum CNR is obtained for H-LSI technique with $\alpha=1$ and $\alpha=3$, respectively. It is basically due to reduced Euclidian distance between constellation points carrying the low priority bits of the HM constellation. An additional penalty of $4.5 \mathrm{~dB}$ is obtained if we compare the performance with a classic 16QAM CR 7/15, which will be the basic transmission mode used to offer global and local services in classic SFN without HM approach. This additional penalty is mainly due to the effect of over modulation used in H-LSI for transmitting the local services. Finally, around of 5 and $8.5 \mathrm{~dB}$ more are required when the local stream is decoded in the TU6 channel model using HM with $\alpha=1$ and $\alpha=3$, respectively.

If decoding of local stream is not fully successful, a second iteration could be carried out, and so on. However, higher number of iteration increases the latency and zapping time of the system.

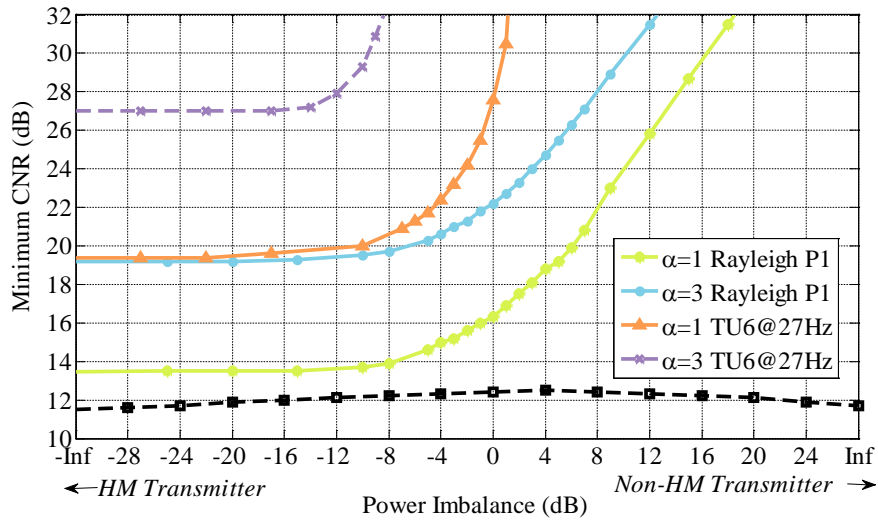

Fig. 8. Performance of local services using H-LSI and ISD decoding. Hierarchical 64QAM CR 7/15 decoding in Rayleigh P1 and TU6 channels. The dashed line is the performance of non-hierarchical 64QAM CR 7/15.

\section{B. O-LSI Coverage Performance}

\section{1) Performance of global services}

With O-LSI, each transmitter inserts local services employing a specific subset of OFDM sub-carriers totally independent of those used for global services. Therefore, the decoding performance of global services is not affected by the insertion of local services. Global services keep all benefits of SFN topologies (i.e. additional coverage by SFN gain) and in this case the coverage depends on the technical characteristics of the base stations, the DVB-NGH transmission mode chosen and the power transmission used. In the overlapping zones between adjacent transmitters not inserting the same local content, global services could experience an SFN gain whereas local services do not.

It should be pointed out that in this approach, the available bitrate for global services is reduced (same MODCOD, but lower number of OFDM sub-carriers) if we want obtain the same coverage level that the conventional SFN approach without insertion of local services. In order to attain the same bitrate for global services a higher MODCOD could be used, reducing the coverage area. However, the total bitrate of the SFN system (global + local services) keeping the same coverage is not reduced; in fact, it could increase as is shown in the section VI.

\section{2) Boosting power for local services}

For O-LSI, the coverage area of local services could basically the same as the coverage of global services without SFN gain, if the same transmission mode is selected. However, the power boosting can be used to increase the coverage area for local services while keeping its available data rate, or increase the transmission capacity for local services while keeping the same coverage level for local and global services.

The maximum power boosting of the local data sub-carriers directly influences the performance of the local services. It depends on the number of LSAs within the SFN, the pilot pattern (PP) and FFT size used. The maximum possible power boosting factor is shown in Fig. 9 represented by the dashed line, assuming that the power that is not used by the unmodulated sub-carries is used by the active data sub-carriers for each transmitter. However, a power correction factor $K$ 


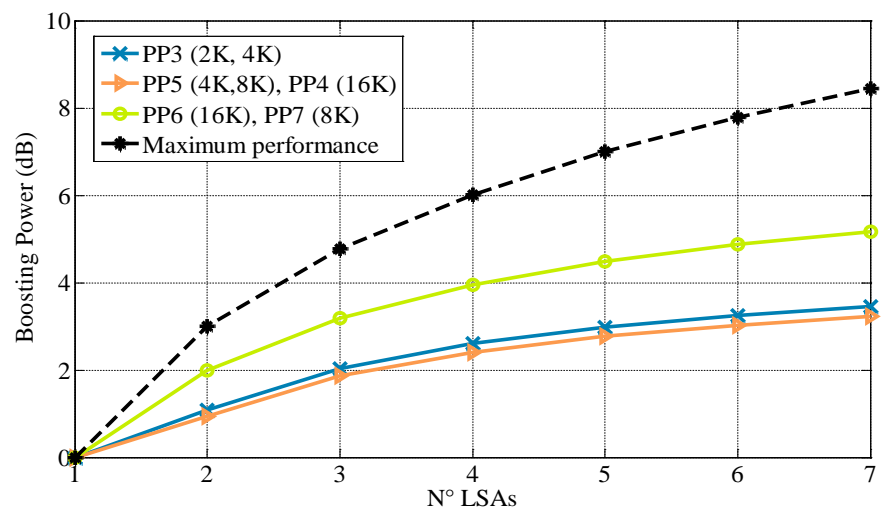

Fig. 9. Power boosting of the O-LSI sub-carriers as a function of the number of LSAs in the SFN. The dashed line is the maximum performance without power correction factor $K$.

must be applied to the power boosting in order to have the same expected average power in the O-LSI starting and closing symbols compared to the preamble P2 symbol. The resulting available boosting power with O-LSI is also shown in Fig. 9. The maximum boosting power (around $5 \mathrm{~dB}$ ) is obtained when PP6 and 16K FFT, or PP7 and 8K FFT are used.

\section{TRANSMISSION CAPACITY RESUltS}

In this section, the capacity gains provided by H-LSI and OLSI are compared to the conventional SFN approach, transmitting the local services across the whole network. It is assumed that one frequency is available for the whole network area for both global and local services. Hence, MFNs are not considered. The capacity gain, $G_{L S I}$, is defined as the ratio between the available data rate for the transmission of global and local services in whole network offered by the new approaches, $R_{L S I}$, and the data rate provided by the conventional SFN approach, $R_{S F N}$ :

$$
G_{L S I}=\frac{R_{L S I}}{R_{S F N}}
$$

The gain depends on the percentage of the data rate of local services relative to the whole data rate of both global and local services $\rho_{\text {local }}$, and the number of local services areas LSAs $n_{L S A}$. For the classic SFN approach, the total available data rate for global and local services is the multiplex data rate $\left(R_{S F N}\right.$ $=R_{\text {Mux }}$ ). In this case, the percentage $\rho_{\text {local }}$ is shared among the different LSAs in the network.

With H-LSI, local services are transmitted on top of global services with HM. Assuming that only Type 1 PLPs are used, the available data rate can be calculated by:

$$
R_{\text {HM-LSI }}=R_{\text {Mux }}\left(1+\frac{\rho_{\text {local }}}{1-\rho_{\text {local }}} \times \frac{D_{X} D_{y}-1}{D_{X} D_{y}}\right),
$$

including the overhead of the local pilot carriers with horizontal spacing $D_{x}$ and vertical spacing $D_{y}$. It should be noted that the maximum percentage of $\rho_{\text {local }}$ for H-LSI assuming the use of all sub-carriers to transmit local content depends on the modulation order and code rates used for the global and local streams, which can be calculated by:

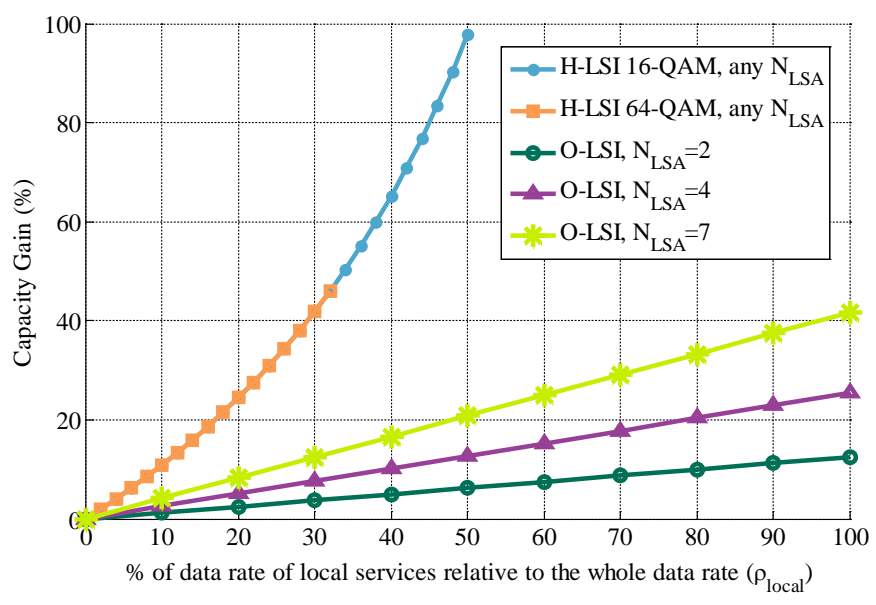

Fig. 10. Capacity gain of HM-LSI and O-LSI as a function of the number of LSAs and the fraction of local services.

$$
\rho_{\text {local, } M a x H-L S I}=\frac{2 \times C R_{\text {local }}}{\left(2+\mu_{\text {global }}\right) \times C R_{\text {global }}},
$$

where $C R_{\text {local }}$ and $C R_{\text {global }}$ are the code rates of the local and global streams, respectively. The parameter $\mu_{\text {global }}$ is the modulation efficiency of the global PLP (e.g. 2 for QPSK and 4 for 16QAM) and the constant factor " 2 " reflects that the local stream is always modulated in QSPK constellation. When the same code rate is used for global and local PLPs, the $\rho_{\text {local }}$ is limited to $50 \%$ and $33.3 \%$ for HM-16QAM and HM64QAM respectively. Fig. 10 shows the potential gains that can be achieved with H-LSI compared to the classic SFN approach as function of $\rho_{\text {local }}$. Both modulations available for H-LSI have been considered (HM-16-QAM and HM-64QAM) with a PP3 $\left(D_{x}=6, D_{x}=4\right)$. It has been assumed that the global and local PLPs employ the same CR, while all OFDM sub-carriers are used to insert local content. As shown in Fig. 10 , it is possible to almost double the available data rate in the network (almost 100\% gain) when seven LSAs are deployed using HM-16QAM, since each HM-16QAM symbol carries two bits of the local stream on top of the two bits of the global stream.

In a similar way to H-LSI, the capacity gain of O-LSI depends on $\rho_{\text {local }}$ and $n_{\text {LSA }}$. Compared to the classic SFN approach, O-LSI provides a capacity gain since it is possible to employ a transmission mode for local services with higher spectral efficiency due to the power boosting of the active carriers. The available data rate with O-LSI can be computed as:

$$
R_{O-L S I}=R_{\text {Mux }}\left(\left(1-\rho_{\text {local }}\right)+\Delta C \times \rho_{\text {local }} \times \frac{D_{X} D_{y}-n_{L S A}-1}{D_{X} D_{y}}\right),
$$

where $\Delta C$ is the capacity gain due to the power boosting. The capacity gain cannot easily be derived because the modulation order and CR enabling the same coverage as the global service depends on the target CNR and the channel model considered. However, assuming that the boosted power level can be directly added to the useful power level of the local PLPs, it is possible to identify a higher modulation order and/or code rate that increases the transmission capacity requiring the same 
CNR as the global PLPs plus the boosted power. Power boosting has been considered as described in the previous section.

Fig. 10 also shows the maximum capacity gain provided by O-LSI for portable reception (Rayleigh P1 channel) compared to the classic SFN approach, using 16QAM as initial modulation scheme for global and local services. The capacity reduction due to the repetition of the scattered pilots for each LSA is included. PP3 and 4K FFT have been chosen in order to obtain the capacity gain with transmission modes suitable for mobile reception. Higher capacity gain could be obtained with the less dense PPs, used mainly for fixed reception, which have high power boosting and low pilot overhead. As shown in the figure, for a possible realistic case with $50 \%$ of local services from all services, O-LSI is more efficient than the classic SFN approach by about $12 \%$ for four local regions or up to $20 \%$ when seven local regions are implemented. The capacity gain increases to $40 \%$ when the MUX is fully dedicated to local services and is shared between seven LSAs, which is the maximum number of LSAs available for O-LSI.

\section{CONCLUSIONS}

The efficient provision of local services in SFNs with minimum increased overhead was one of the commercial requirements underlying DVB-NGH. This paper has analyzed the implementation issues and evaluated the performance in terms of minimum CNR required for successful decoding and capacity gain of the two complementary technical solutions adopted, known as H-LSI (based on hierarchical modulation) and O-LSI (using orthogonal transmission mode). Either technique addresses different use cases with a different coverage/capacity performance trade-off. The optimum transmission technique depends on the target use case and the particular scenario considered (location and power of the transmitters, distribution of the LSAs, etc.). Both solutions preserve the SFN advantage for global services by broadcasting the local content in their respective target areas only, avoiding SFN self-interference between them.

Compared with the classic SFN approach, H-LSI offers a high capacity gain for local services while keeping the data rate of the global stream constant, at the expense of a coverage reduction for both global and local services. O-LSI provides local services potentially with the same coverage as the global services with a moderate transmission capacity gain at the expense of reducing the available data rate for global services.

\section{ACKNOWLEDGEMENT}

The authors thank their colleagues at iTEAM of Universitat Politècnicade València and Institut fuer Nachrichtentechnik of Technische Universitaet Braunschweig, especially Prof. Dr.Ing. Ulrich Reimers and Prof. Dr.-Ing. Narcis Cardona, for the helpful suggestions and comments. Furthermore, they are grateful to Frank Herrmann and all other colleagues of the DVB-NGH development team for the fruitful discussions.

\section{REFERENCES}

[1] W. Joseph, P. Angueira, J. A. Arenas, L. Verloock, and L. Martens, "On the Methodology for Calculating SFN Gain in Digital Broadcast Systems," IEEE Trans. on Broadcasting, vol. 56, no. 2, pp. 331-339, Sept. 2010.

[2] D. Gómez-Barquero, "Next Generation Mobile Broadcasting - An Overview of the Next-Generation Mobile Digital Video Broadcasting Standard DVB-NGH," CRC press, pp. 185-221, 2013.

[3] EN 302755 v1.3.1, "Frame Structure Channel Coding and Modulation for a Second Generation Digital Terrestrial Television Broadcasting System (DVB-T2),” Nov. 2011.

[4] J. Zöllner, J. López-Sánchez, D. Gómez-Barquero, S. Atungsiri, and E. Stare, "Next Generation Mobile Broadcasting - Local Service Insertion in DVB-NGH Single-Frequency Networks," CRC press, pp. 513-548, 2013.

[5] H. Jiang, P. Wilford, S. Wilkus: Providing Local Content in a Hybrid Single Frequency Network Using Hierarchical Modulation, IEEE Trans. on Broadcasting, vol. 56, pp. 532-540, 2010.

[6] Hong Jiang; Wilford, P.A., "A hierarchical modulation for upgrading digital broadcast systems," IEEE Transactions on Broadcasting, vol.51, no.2, pp.223,229, June 2005

[7] ETSI Std. EN 300 744, Rev. 1.6.1, "Framing structure, channel coding and modulation for digital terrestrial television”, 2009.

[8] M. R. Chari et al., "FLO Physical Layer: An Overview," IEEE Trans. on Broadcasting, vol. 52., no. 1, pp. 145-160, March 2007.

[9] ETSI EN 302583 v1.1.2, "Digital Video Broadcasting (DVB); Framing Structure, Channel Coding and Modulation for Satellite Services to Handheld devices (SH) below 3G,” Feb, 2010.

[10] ETSI TS 102992 v1.1.1, "Structure and modulation of optional transmitter signatures (T2-TX-SIG) for use with the DVB-T2 second generation digital terrestrial television broadcasting system," 2010.

[11] J. Zöllner, J. Robert, S. Atungsiri, and M. Taylor, "Local Service Insertion in Terrestrial Single Frequency Networks Based on Hierarchical Modulation," Proc. IEEE International Conference on Consumer Electronics (ICCE), Las Vegas, USA, 2012.

[12] Next Generation Broadcasting System to Handheld, Physical Layer Specification (DVB-NGH), DVB BlueBook A160, DVB Std.,2012.

[13] Boveda, J.; Marcos, G.; Perez, J.M.; Ponce, S.; Aranaz, A., "MER degradation in a broadcast mobile network," Broadband Multimedia Systems and Broadcasting, 2009. BMSB '09. IEEE International Symposium on, pp.1,5, 13-15 May 2009.

[14] Keqian Yan; Fang Yang; Changyong Pan; Jian Song, "Reception Quality Prediction in a Single Frequency Network for the DTMB Standard," IEEE Trans. on Broadcasting, vol.58, no.4, pp.629,636, Dec. 2012.

[15] Rico-Alvarino, A.; Mosquera, C.; Perez-Gonzalez, F., "Overlay Cognitive Transmission in a Multicarrier Broadcast Network with Dominant Line of Sight Reception,"IEEE Transactions on Wireless Communications, vol.11, no.11, pp.4128,4139, November 2012. 